\title{
Efficacy of Intensive Neurodevelopmental Treatment for Children With Developmental Delay, With or Without Cerebral Palsy
}

\author{
Kyoung Hwan Lee, MD, Jin Woo Park, MD, Ho Jun Lee, MD, Ki Yeun Nam, MD, \\ Tae June Park, MD, Hee Jae Kim, MD, Bum Sun Kwon, MD
}

Department of Physical Medicine and Rehabilitation, Dongguk University College of Medicine, Goyang, Korea

\begin{abstract}
Objective To evaluate the effectiveness of intensive neurodevelopmental treatment (NDT) on gross motor function for the children having developmental delay (DD), with or without cerebral palsy (CP).

Methods Forty-two children had intensive NDT three times weekly, 60 minutes a day, for 3 months, immediately followed by conventional NDT once or twice a week, 30 minutes a day, for another 3 months. We assessed Gross Motor Function Measure (GMFM) over three time points: before conventional NDT, before and after intensive NDT, and after 3 months of additional conventional NDT.

Results The GMFM score in DD children significantly improved after intensive NDT, and the improvement maintained after 3 months of conventional NDT $(\mathrm{p}<0.05)$. The children were further divided into two groups: DD with $\mathrm{CP}$ and DD without $\mathrm{CP}$. Both groups showed significant improvement and maintained the improvements, after intensive NDT $(\mathrm{p}<0.05)$. Also, there was no significant difference in treatment efficacy between the two groups. When we calculate the absence rate for comparing the compliance between intensive and conventional NDT, the absence rate was lower during the intensive NDT.

Conclusion Intensive NDT showed significantly improved gross motor function and higher compliance than conventional NDT. Additionally, all improvements were maintained through subsequent short-term conventional NDT. Thus, we recommend the intensive NDT program by day-hospital centers for children with DD, irrespective of accompanying CP.
\end{abstract}

Keywords Cerebral palsy, Developmental disabilities, Disability evaluation, Rehabilitation

Received May 23, 2016; Accepted August 22, 2016

Corresponding author: Bum Sun Kwon

Department of Physical Medicine and Rehabilitation, Dongguk University College of Medicine, 27 Dongguk-ro, Ilsandong-gu, Goyang 10326, Korea. Tel: +82-31-961-7460, Fax: +82-31-961-7488, E-mail: bskwon@dumc.or.kr

ORCID: Kyoung Hwan Lee (http://orcid.org/0000-0002-8720-3315); Jin Woo Park (http://orcid.org/0000-0003-4989-2575); Ho Jun Lee (http://orcid. org/0000-0002-1997-2593); Ki Yeun Nam (http://orcid.org/0000-0001-6932-6541); Tae June Park (http://orcid.org/0000-0001-7166-059X); Hee Jae Kim (http://orcid.org/0000-0001-6062-5246); Bum Sun Kwon (http://orcid.org/0000-0001-7755-435X).

(a) This is an open-access article distributed under the terms of the Creative Commons Attribution Non-Commercial License (http://creativecommons.org/ licenses/by-nc/4.0) which permits unrestricted noncommercial use, distribution, and reproduction in any medium, provided the original work is properly cited. Copyright $\odot 2017$ by Korean Academy of Rehabilitation Medicine 


\section{INTRODUCTION}

Cerebral palsy (CP) is defined as a non-progressive neurological disorder resulting in movement and posture disability [1]. The treatment goal of $\mathrm{CP}$ is to improve the motor function through physical therapy, including muscle strengthening, endurance exercise, and range of joint motion exercise [2,3]. Various methods of physical therapy are known for CP children [4]. Among these, neurodevelopmental treatment (NDT) has achieved global acceptance since 1940 [5-7]. Based on a reflex/hierarchical model, NDT inhibits the abnormal movements and promotes normal movements by stimulating key points of motion, and also emphasizes in developing automatic righting reaction [2]. Nowadays, NDT is widely used to treat CP patients [8], and also has a positive effect in patients with developmental delay (DD), other than CP [9]. DD may be manifested by various causes; it is a condition defined when a child has not achieved skills such as cognitive, physical and emotional development, communication and adaptive behavior, lagging by 2 standard deviation in at least one skill, or by 1.5 standard deviation in at least two skills mentioned above [10]. Various rehabilitation programs are required to enhance the development, and current practices include NDT.

To standardize NDT, medical doctors and physical therapist are educated every year. Thus, the qualitative aspects of the treatment are managed by certification of NDT education. However, the quantitative aspects (focusing on the frequency or duration of NDT) remain inconclusive, although these factors determine the intensity of NDT $[11,12]$. For this reason, the intensity of treatment tends to depend on the individual hospital settings or the familial support for patients. In Korea, two regimens of NDT are generally provided at the out-patient and in-patient departments (or day-hospital center). For out-patients, conventional NDT comprising of lesser frequency (once or twice a week) and short duration (30 minutes or less a session) is provided. The in-patients are provided intensive NDT, which is more frequent ( 3 to 5 times a week) and of a longer duration (30 to 60 minutes per a session). Some studies addressed the tendency toward greater improvement by the intensive treatment $[13,14]$. However, there is no compelling evidence on which regimens of NDT are more effective, because of the great variation in age and severity of CP. Furthermore, a consensus to consistent regimen for intensive NDT is yet to be established. One study reported the effectiveness of intensive physiotherapy at 4 times a week over 4 weeks [14]. Another study reported an individualized intermittent intensive physical therapy schedule for a child with spastic quadriplegia, and revealed the highest increment in mean score of the Gross Motor Function Measure (GMFM-66) obtained during the 5 times a week of intensive therapy period, compared to the 2 times a week of conventional therapy [15]. A randomized controlled crossover study showed the effectiveness of intensive physical therapy in infants with cerebral palsy in the area of sitting acquisition [16]. However, these studies of intensive physical therapy are pilot studies with a small number of patients of DD with CP, and have never been attempted in DD patients without CP.

We therefore evaluated the effectiveness of intensive NDT for patients of DD, with or without CP. We applied conventional NDT after intensive NDT, and tracked the gross motor function changes to see whether the effect of intensive NDT were maintained. We also investigated the compliance of intensive NDT by comparing the number of treatment days cancelled during intensive NDT and conventional NDT.

\section{MATERIALS AND METHODS}

\section{Participants}

Retrospective chart review was conducted for the patients who had undergone day hospital center rehabilitation program in Dongguk University Hospital, Korea, from March 2010 to December 2014. A total of 69 patients were screened; they had DD according to the developmental test, including a diagnosis of CP; all of them had been treated with conventional NDT during out-patient rehabilitation program. Among these, we selected the patients who showed little improvement by conventional NDT. We defined 'little improvement' when there is a decrease or no change of GMFM score during the outpatient rehabilitation program. Patients who did not complete the 3 months of day-hospital center rehabilitation program, and had not extended the 3 months of outpatient rehabilitation program immediately after dayhospital center, were excluded. We also excluded the patients who had orthopedic surgery for articular or spinal deformity, botulinum toxin injection and any acute 
medical condition (such as seizure and pneumonia) 3 months prior to the treatment, since this would affect the score of functional assessment. Patients were similarly excluded by the same criteria during the next 6 -month period of day-hospital center and out-patient. Finally, 42 patients with DD were enrolled (Fig. 1). The mean age of total subjects was $59.88 \pm 22.58$ months, and male/female ratio was $25 / 17$. Of these, 24 patients ( 14 males and 10 females) were DD with $\mathrm{CP}$, and 18 patients (11 males and 7 females) were DD without CP. The mean age of DD with $\mathrm{CP}$ was $68.25 \pm 22.18$ months, and that of DD without $\mathrm{CP}$ was $48.73 \pm 18.47$ months. Clinical features of DD for both groups are given below (Table 1). When we divide the children by the Gross Motor Function Classification System (GMFCS), there was no significant difference among GMFCS levels in both DD with or without CP. The study was approved by the Institutional Review Board of Dongguk University Hospital.

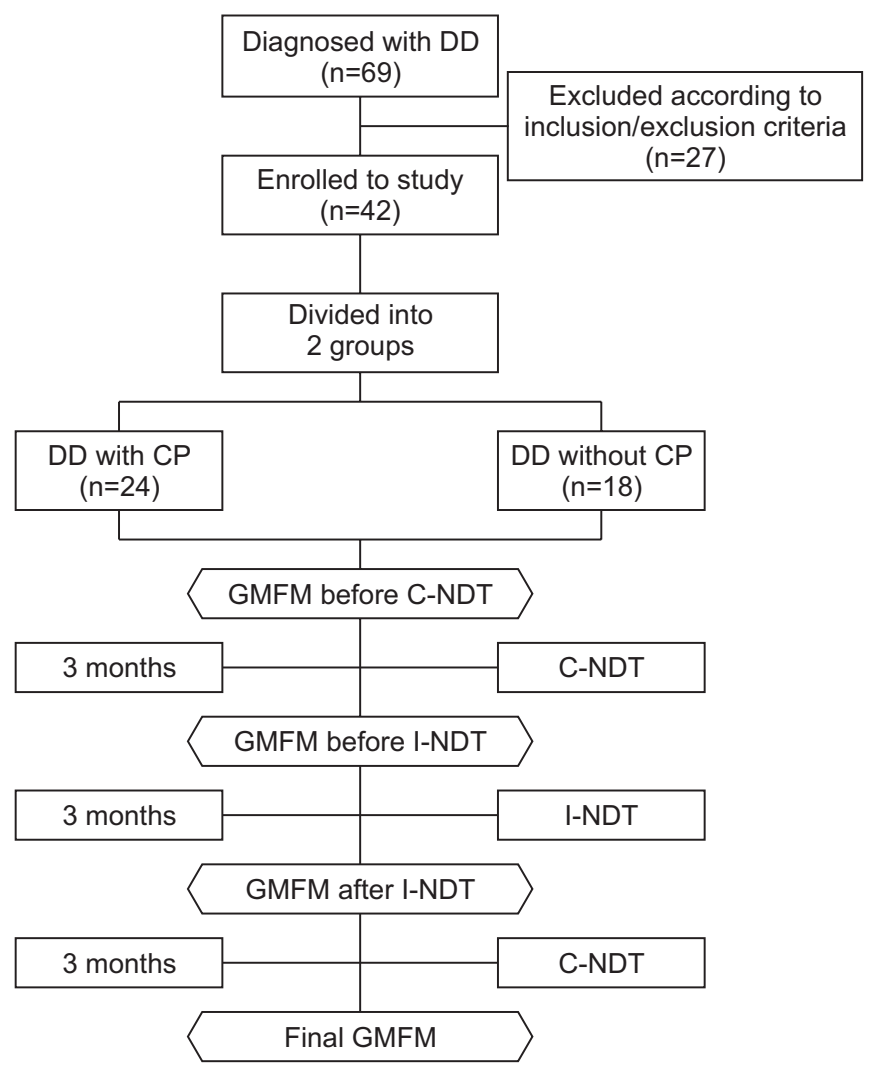

Fig. 1. Research design and flow. DD, developmental delay; CP, cerebral palsy; GMFM, Gross Motor Function Measure; C-NDT, conventional neurodevelopmental treatment; INDT, intensive neurodevelopmental treatment.

\section{Intervention}

In Dongguk University Hospital, we have two programs for rehabilitation of DD patients, with or without CP: out-patient and day-hospital center protocol. For the out-patient protocol, NDT is once or twice a week for 30 minutes a day, and is defined as conventional NDT. For the day-hospital center protocol, NDT is thrice weekly for 60 minutes a day, and is defined as intensive NDT. Occupational therapy, speech therapy and functional activities are also provided for patients of both out-patient and day-hospital center. Each child has a designated therapist who underwent the intervention goal setting and treatment in accordance with the principles of NDT. Since all designated therapists are certified for NDT, we could minimize the risk of personal deviation whilst conducting the procedure.

Table 1. Clinical features for the children of developmental delay (DD) with or without CP

\begin{tabular}{lcc}
\hline \multicolumn{1}{|c}{ Variable } & $\begin{array}{c}\text { DD } \\
\text { with CP } \\
(\mathbf{n = 2 4 )}\end{array}$ & $\begin{array}{c}\text { DD } \\
\text { without CP } \\
(\mathbf{n = 1 8})\end{array}$ \\
\hline Clinical feature & $16(66.7)$ & - \\
\hline Spastic bilateral & $8(33.3)$ & - \\
\hline $\begin{array}{l}\text { Spastic unilateral } \\
\text { Diagnosis } \\
\text { Congenital brain anomaly }\end{array}$ & - & $5(27.7)$ \\
\hline Arnold-Chiari malformation & - & 1 \\
\hline Dandy-Walker syndrome & - & 1 \\
\hline Partial agenesis of corpus & - & 1 \\
$\quad$ callosum & & \\
\hline Microcephaly & - & 2 \\
\hline Genetic disorder & - & $3(16.7)$ \\
\hline CHARGE syndrome & - & 1 \\
\hline Prader Willi syndrome & - & 1 \\
\hline Mitochondrial disease & - & 1 \\
\hline Unspecified & - & $10(55.6)$ \\
\hline GMFCS & & \\
\hline I & $6(25.0)$ & $3(16.7)$ \\
\hline II & $4(16.7)$ & $2(11.1)$ \\
\hline III & $5(20.8)$ & $7(38.8)$ \\
\hline IV & $3(12.5)$ & $3(16.7)$ \\
\hline V & $6(25.0)$ & $3(16.7)$ \\
\hline
\end{tabular}

Values are presented as number of patients (\%).

CP, cerebral palsy; GMFCS, Gross Motor Function Classification System. 


\section{Outcome measures}

Assessments were made before conventional NDT, before and after intensive NDT, and after conventional NDT, by the GMFM. GMFM is a standardized method for evaluating gross motor function of CP children, and many studies have proved its reliability and validation [17-19]. GMFM was done by the physical therapists who were not providing the conventional and intensive NDT to the patients. The flow of NDT application and GMFM assessment is shown diagrammatically (Fig. 1). All patients were treated with conventional NDT for 3 months. Before the first conventional NDT treatment, we obtained 'GMFM before C-NDT'. Before starting the dayhospital center, we obtained 'GMFM before intensive NDT'. Immediately after 3 months of day-hospital center rehabilitation program, 'GMFM after intensive NDT' was obtained. At the end of 3 months of the conventional NDT in the out-patient, 'Final GMFM' was obtained. All GMFMs from both groups were compared.

The absence rate was calculated by the percentage (\%) of number of days which children did not attend the treatment sessions among reservation days during intensive NDT and conventional NDT period, and compared between conventional and intensive NDT period to evaluate compliance of treatment.

\section{Statistical analysis}

Statistical analyses were performed with SPSS-K ver. 20.0 (IBM SPSS, Armonk, NY, USA). In order to compare the differences among GMFM scores and verify the effectiveness of intensive NDT according to the time flow, repeated measured ANOVA was used. Patients were divided into DD with $\mathrm{CP}$ group and DD without $\mathrm{CP}$ group. We evaluated whether intensive NDT had the equivalent effect on both groups by using the same statistical method. A p-value $<0.05$ was considered statistically signifi- cant; confidence interval of $95 \%$ was used.

\section{RESULTS}

Changes of GMFM scores over the three periods

The mean GMFM score before the first conventional NDT was $58.03 \pm 23.37$, which was not a statistically significant difference with GMFM before intensive NDT (57.58 \pm 23.62$)$. 'GMFM score before intensive NDT' increased from $57.58 \pm 23.62$ to $61.76 \pm 23.71$ after intensive NDT (Table 2), which was a statistically significant improvement $(\mathrm{p}<0.05)$. After the conventional NDT, 'Final GMFM' was statistically not different from 'GMFM after intensive NDT', which revealed that the improvement after intensive NDT was maintained during 3 months of conventional NDT (Table 2).

When we divided the children into DD with CP group and DD without CP group, the changes of GMFM score before and after intensive NDT showed a statistically significant improvement along with maintenance of the effect $(p<0.05)$. However, the changes of GMFM after intensive and conventional NDT revealed no differences $(\mathrm{p}=0.582)$ between the two groups. When we divide the children by GMFCS or ages in various aspects, there was no significant difference among the parameters in both groups.

\section{Absence rate during treatment \\ Reservation was cancelled 46 times out of 1,764 during the conventional NDT period, and absence rate was $2.6 \%$. Correspondingly, reservation was cancelled 18 times out of 1,512 during the intensive NDT period, and absence rate was $1.2 \%$, which was lower than that of conventional NDT.}

Table 2. The changes of GMFM score before and after neurodevelopmental treatment

\begin{tabular}{lccc}
\hline & DD with CP & DD without CP & Total \\
\hline GMFM before C-NDT & $55.32 \pm 25.42$ & $61.65 \pm 18.58$ & $58.03 \pm 23.37$ \\
GMFM before I-NDT & $54.82 \pm 26.21$ & $61.26 \pm 19.02$ & $57.58 \pm 23.62$ \\
GMFM after I-NDT & $58.63 \pm 26.56$ & $65.94 \pm 18.95$ & $61.76 \pm 23.71$ \\
Final GMFM & $58.51 \pm 27.11$ & $65.73 \pm 18.39$ & $61.60 \pm 23.79$ \\
\hline
\end{tabular}

Values are presented as mean \pm standard deviation. GMFM, Gross Motor Function Measure; DD, developmental delay; CP, cerebral palsy; C-NDT, conventional neurodevelopmental treatment; I-NDT, intensive neurodevelopmental treatment. 


\section{DISCUSSION}

Cerebral palsy results limitation of activity, accompanied by impairment of sensory, cognition, communication, and perception [1]. Different factors of treatment, such as when to start the initial treatment, and what kind of treatment is more effective, have been inconclusive. However, early treatment helps in reconstructing the damaged brain, and is likely to improve the activity functions. It is proven that rapid growth and development of the brain occurs during infancy, and that interaction with environment plays an important role; hence, an early treatment is worth considering and should be applied intensively [2].

When the concept of NDT was suggested in 1940, clinical observation was thought to be critical to determine the effectiveness of the treatment as the mechanism of central nervous system development had not been established, and evidence-based medicine had not been generally accepted. For this reason, although NDT had weak evidence for regular application and quantification of frequency or intensity, it made the fundamental basis of $\mathrm{CP}$ treatment.

Intensive NDT is a concept that promotes a greater therapeutic effect, by increasing the frequency and duration of the treatment. As mentioned in some reports [13-15], the outcomes of this study also supports similar results that efficacy of NDT improved when increased frequency and duration was applied. In this study, we applied intensive NDT to patients who had little improvement by conventional NDT, and we set 'little improvement' to a decrease or no change of GMFM score. The mean age of the enrolled patients was $59.58 \pm 22.77$ months, which is the time when GMFM shows little change according to the predicted average development by the GMFM in CP [20]. Further, we excluded the patients who had any acute medical conditions.

The advantage of intensive NDT was reported to diminish over the subsequent 6 months [13]. In this study, we followed GMFM score after intensive treatment and found that the improvement of GMFM was maintained during 3 months of conservative treatment. Additionally, the efficacy of intensive NDT was found not only in group of DD with CP, but also in DD without CP. Very few studies have reported the achievability of NDT when its intensity and frequency were increased in children with
DD. Therefore, our study has great significance in determining the effectiveness of NDT in DD, as well as CP.

Before this study, we hypothesized that the children having DD with $\mathrm{CP}$ had spasticity in the limbs, and would therefore be unable to maintain the effect of intensive NDT after they were switched to conventional NDT. Meanwhile, children having DD without CP were expected to maintain or increase their GMFM score after intensive NDT followed by conventional NDT, since spasticity would not be a concern. However, results showed that intensive NDT was effective not only in DD without CP, but also in DD with CP, and the effects were maintained in both groups after the conventional NDT.

An earlier study had shown NDT at four times a week for 4 weeks, followed by a rest phase of 8 weeks, resulted in increment of GMFM [14], whereas NDT at 4 times a week for 6 weeks followed by rest phase of 6 weeks also achieved increment of GMFM [21]. In our study, we skipped the rest phase, but applied conventional NDT later. Because most patients and their parents wanted the treatment to be ongoing, it was not ethical to have a rest phase for clinical trial. Furthermore, we could not assign a patient to intensive or conventional NDT group randomly because of the possible ethical issue as well.

Reviewing all the GMFM scores from the out-patient

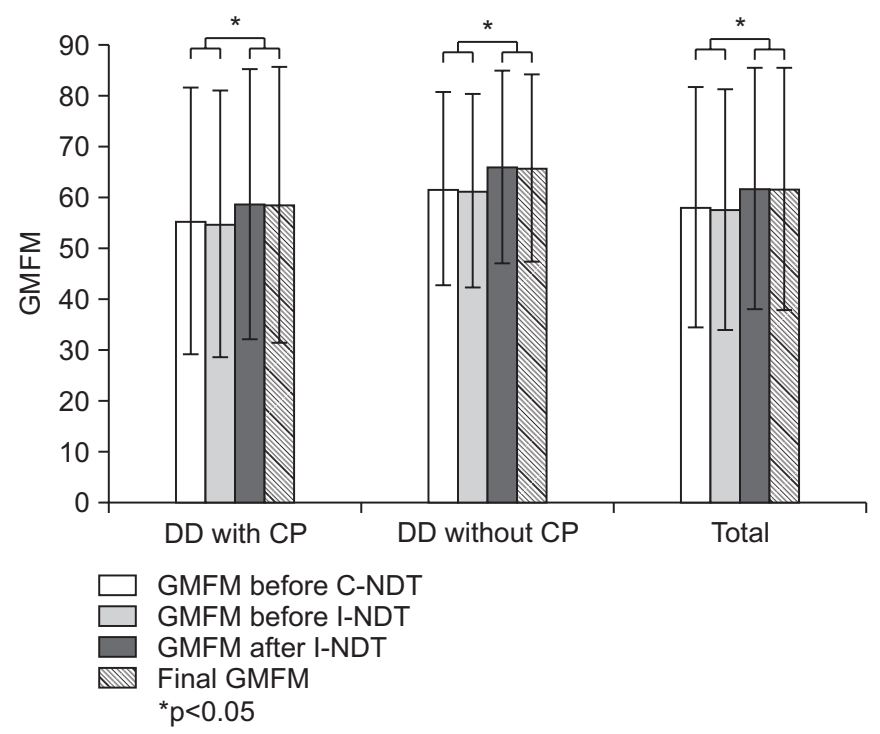

Fig. 2. Comparison of GMFM scores over the four periods. $\mathrm{CP}$, cerebral palsy; DD, developmental delay; GMFM, Gross Motor Function Measure; C-NDT, conventional neurodevelopmental treatment; I-NDT, intensive neurodevelopmental treatment. 
before day hospital center, we found that the final GMFM also showed significant improvement compared to GMFM during the out-patient before day hospital center (Fig. 2). Thus, we recommend intensive NDT intermittently or periodically when the out-patient treatment is not effective anymore and boring for the patient.

This study has several limitations; we did not set a control group with complete treatment by conventional NDT for the entire period. It was difficult to secure sufficient number of patients satisfying the criteria with random assignment. We did not set two types of sessions alternately in all screened children by 'ABAB' comparison since we were unable to control other medical problems for a long period, and could not match the period of final 'B' treatment. Many patients were excluded during the study due to several conditions that might affect the gross motor functions, such as pneumonia, unscheduled surgery, or botulinum toxin injection. Another limitation is that we did not control the time and frequency of occupation therapy and functional activity training programs, although the treatments were applied during both intensive and conventional NDT periods. Many therapists applied the concept of NDT in occupation therapy and functional training, and these treatments could affect the GMFM score. Thus, further evaluations for various other standardized tools are needed in future studies, such as the upper arm function or activity of daily living, to investigate the effect of intensive NDT more specifically. Lastly, we observed the effect of intensive NDT was maintained for only 3 months, even though a previous report had been published with a follow-up for 18 months in CP [12]. Our study is considered significant in that a 3-month effect of intensive NDT was maintained in children with DD without $\mathrm{CP}$, and it confirms the shortterm maintenance of intensive NDT. Therefore, further follow-up studies should be conducted at our institution to verify the long-term effects.

In conclusion, GMFM scores significantly improved in both DD with and without CP children with 3 months of intensive NDT, and the effect was maintained after conventional NDT. Absence rate was relatively lower in intensive NDT session, which implies better concentration and compliance to intensive NDT resulting in feasibility of intensive NDT. Our results support that we could recommend the short-term intensive NDT program to in-patient or day-hospital center for all DD children, ir- respective of their CP status.

\section{CONFLICT OF INTEREST}

No potential conflict of interest relevant to this article was reported.

\section{REFERENCES}

1. Lim SY, Jung HY. Cerebral palsy: etiology and evaluation. In: Sung IY, editor. Pediatric rehabilitation. 2nd ed. Seoul: Koonja; 2013. p. 367-82.

2. Sung IY, Shin YB, Park SS. Cerebral palsy: clinical features and management. In: Sung IY, editor. Pediatric rehabilitation. 2nd ed. Seoul: Koonja; 2013.p. 383-418.

3. Barry MJ. Physical therapy interventions for patients with movement disorders due to cerebral palsy. J Child Neurol 1996;11 Suppl 1:S51-60.

4. Molnar GE, Alexander MA. Pediatric rehabilitation. 3rd ed. Philadelphia: Hanley \& Belfus; 1999.

5. Bobath B. A neuro-developmental treatment of cerebral palsy. Physiotherapy 1963;49:242-4.

6. Bobath B. Motor development, its effect on general development, and application to the treatment of cerebral palsy. Physiotherapy 1971;57:526-32.

7. Tsorlakis N, Evaggelinou C, Grouios G, Tsorbatzoudis C. Effect of intensive neurodevelopmental treatment in gross motor function of children with cerebral palsy. Dev Med Child Neurol 2004;46:740-5.

8. Kim SJ, Lee CH, Na JK, Lee SH, Sung IY, Park BK. Current physical therapy status for the children with cerebral palsy in Korea. J Korean Acad Rehabil Med 2000;24:200-7.

9. Ottenbacher KJ, Biocca Z, DeCremer G, Gevelinger M, Jedlovec KB, Johnson MB. Quantitative analysis of the effectiveness of pediatric therapy: emphasis on the neurodevelopmental treatment approach. Phys Ther 1986;66:1095-101.

10. Jung HY. Functional assessment of pediatric disabilities. In: Sung IY, editor. Pediatric rehabilitation. 2nd ed. Seoul: Koonja; 2013. p. 59-84.

11. Blauw-Hospers CH, Hadders-Algra M. A systematic review of the effects of early intervention on motor development. Dev Med Child Neurol 2005;47:421-32.

12. Siebes RC, Wijnroks L, Vermeer A. Qualitative analysis of therapeutic motor intervention programmes 
for children with cerebral palsy: an update. Dev Med Child Neurol 2002;44:593-603.

13. Bower E, Michell D, Burnett M, Campbell MJ, McLellan DL. Randomized controlled trial of physiotherapy in 56 children with cerebral palsy followed for 18 months. Dev Med Child Neurol 2001;43:4-15.

14. Trahan J, Malouin F. Intermittent intensive physiotherapy in children with cerebral palsy: a pilot study. Dev Med Child Neurol 2002;44:233-9.

15. Rahlin M. An individualized intermittent intensive physical therapy schedule for a child with spastic quadriparesis. Physiother Theory Pract 2011;27:51220.

16. Shamir M, Dickstein R, Tirosh E. Intensive intermittent physical therapy in infants with cerebral palsy: a randomized controlled pilot study. Isr Med Assoc J 2012;14:737-41.

17. Palisano RJ, Hanna SE, Rosenbaum PL, Russell DJ, Walter SD, Wood EP, et al. Validation of a model of gross motor function for children with cerebral palsy. Phys Ther 2000;80:974-85.

18. Palisano RJ, Walter SD, Russell DJ, Rosenbaum PL, Gemus M, Galuppi BE, et al. Gross motor function of children with down syndrome: creation of motor growth curves. Arch Phys Med Rehabil 2001;82:494500.

19. Russell DJ, Avery LM, Rosenbaum PL, Raina PS, Walter SD, Palisano RJ. Improved scaling of the gross motor function measure for children with cerebral palsy: evidence of reliability and validity. Phys Ther 2000;80:873-85.

20. Rosenbaum PL, Walter SD, Hanna SE, Palisano RJ, Russell DJ, Raina P, et al. Prognosis for gross motor function in cerebral palsy: creation of motor development curves. JAMA 2002;288:1357-63.

21. Christiansen AS, Lange C. Intermittent versus continuous physiotherapy in children with cerebral palsy. Dev Med Child Neurol 2008;50:290-3. 